\title{
Jordanian Twist Quantization of $D=4$ Lorentz and Poincaré Algebras and $D=3$ Contraction Limit
}

\author{
A. Borowiec ${ }^{1)}$, J. Lukierski ${ }^{1)}$ and V.N. Tolstoy ${ }^{1), 2)}$ \\ 1) Institute for Theoretical Physics, \\ University of Wrocław, pl. Maxa Borna 9, \\ 50-205 Wrocław, Poland \\ ${ }^{2)}$ Institute of Nuclear Physics, \\ Moscow State University, 119992 Moscow, Russia
}

\begin{abstract}
We describe in detail two-parameter nonstandard quantum deformation of $D=4$ Lorentz algebra $\mathfrak{o}(3,1)$, linked with Jordanian deformation of $\mathfrak{s l}(2 ; \mathbb{C})$. Using twist quantization technique we obtain the explicit formulae for the deformed coproducts and antipodes. Further extending the considered deformation to the $D=4$ Poincaré algebra we obtain a new Hopf-algebraic deformation of four-dimensional relativistic symmetries with dimensionless deformation parameter. Finally, we interpret $\mathfrak{o}(3,1)$ as the $D=3$ de-Sitter algebra and calculate the contraction limit $R \rightarrow \infty(R$-deSitter radius) providing explicit Hopf algebra structure for the quantum deformation of the $D=3$ Poincaré algebra (with masslike deformation parameters), which is the two-parameter light-cone $\kappa$-deformation of the $D=3$ Poincaré symmetry.
\end{abstract}

\section{Introduction}

In the last decade there were considered quantum field theories on noncommutative spacetime, after it has been shown that the noncommutativity might follow from quantum gravity corrections (see e.g. [1]) or an open string theory with anti-symmetric tensor field background (see e.g. [2]). For the simple examples of noncommutativity, in particular Heisenberg-like space-time commutators $\left(\theta_{\mu \nu}=\right.$ const $)$ it has been shown $[3,4,5,6,7]$ that the theory with noncommutative space-time is covariant under twisted Poincaré symmetry. In such approach the violation of classical Poincaré invariance, e.g., by constant tensor $\theta_{\mu \nu}$, can be equivalently described by twisting of classical symmetry [6]. Because twist quantizations of the Lorentz and Poincaré algebras are classified by classical $r$ matrices satisfying homogeneous Yang-Baxter (YB) equations it is interesting to approach the problem of possible violations of Lorentz and Poincaré symmetries by considering new, in particular non-Abelian, twist quantizations ${ }^{1}$.

\footnotetext{
${ }^{1}$ By non-Abelian twist we mean a twist two-tensor with a support in a non-Abelian algebra.
} 
All quantum deformations of relativistic symmetries are described by Hopf-algebraic deformations of Lorentz and Poincaré algebras. Such quantum deformations determine infinitesimally Lorentz and Poincaré Poisson structures. These Poisson structures are described by classical $r$-matrices satisfying homogeneous as well as inhomogeneous (modified) YB equations and they were classified already some time ago by S. Zakrzewski (see [8] for the Lorentz classical $r$-matrices and [9] for the Poincaré classical matrices). In [8] there are provided four classical $\mathfrak{o}(3,1) r$-matrices and in [9] one finds 21 cases describing different deformations of Poincaré symmetry, with various numbers of free parameters.

In this paper we would like to describe the explicit Hopf algebra for an important nonstandard deformation of the $D=4$ Lorentz algebra generated by two-parameter Jordanian classical $r$-matrix.

Part of the results presented in this paper has been given in our short report [10] where the complex algebra basis was used. In contrast to [10] here all obtained results are represented in real (more physical) basis of the Lorentz algebra. In this case a twist twotensor depends on new generators $\sigma$ and $\varphi$ which are operator analogs of polar coordinates for a complex plane. Moreover we interpret $D=4$ Lorentz algebra as $D=3$ de-Sitter algebra and following the quantum contraction method applied firstly to the $q$-deformed $D=4$ anti-de-Sitter (dS) algebra [11] we calculate the contraction limit $R \rightarrow \infty(R$ - de-Sitter radius) providing a deformation of $D=3$ Poincaré algebra. Subsequently, we present in explicit form the Hopf structure describing two-parameter extension of the light-cone $\kappa$-deformation for $D=3$ Poincaré symmetry.

The plan of the paper is the following. In Sect.2 we describe different bases of the real Lorentz algebra, $\mathfrak{o}(3,1)$, and recall the classification of all possible quantum deformations of the $D=4$ Lorentz algebra [9]. In Sect.3 we calculate the twist function corresponding to the non-Abelian Jordanian classical $r$-matrix (2.17) and describe explicitly the deformed coproducts and antipodes in classical $\mathfrak{o}(3,1)$ basis. In Sect. 4 we obtain a deformed $D=4$ Poincaré-Hopf algebra by adding four momentum generators and calculating their twisted coproducts. In Sect.5 we rewrite our quantum deformation in terms of $D=3$ de-Sitter algebra and we perform the contraction, providing two-parameter light cone $\kappa$-deformation of the $D=3$ Poincaré symmetry. In Sect.6 we present possible extensions of the present work.

\section{$2 \quad D=4$ Lorentz algebra and its classical $r$-matrices}

The classical canonical basis of the $D=4$ Lorentz algebra, $\mathfrak{o}(3,1)$, can be described by anti-Hermitian six generators $\left(h, e_{ \pm}, h^{\prime}, e_{ \pm}^{\prime}\right)$ satisfying the following nonvanishing commutation relations:

$$
\begin{gathered}
{\left[h, e_{ \pm}\right]= \pm e_{ \pm}, \quad\left[e_{+}, e_{-}\right]=2 h} \\
{\left[h, e_{ \pm}^{\prime}\right]= \pm e_{ \pm}^{\prime}, \quad\left[h^{\prime}, e_{ \pm}\right]= \pm e_{ \pm}^{\prime}, \quad\left[e_{ \pm}, e_{\mp}^{\prime}\right]= \pm 2 h^{\prime}} \\
{\left[h^{\prime}, e_{ \pm}^{\prime}\right]=\mp e_{ \pm}, \quad\left[e_{+}^{\prime}, e_{-}^{\prime}\right]=-2 h}
\end{gathered}
$$

and moreover

$$
x^{*}=-x \quad(\forall x \in \mathfrak{o}(3,1)) .
$$


The formulas (2.1)-(2.3) can be rewritten as the following three $\mathfrak{o}(3)$-covariant relations describing Lorentz algebra in "physical" basis $(i, j, k=1,2,3)^{2}$

$$
\left[M_{i}, M_{j}\right]=\imath \epsilon_{i j k} M_{k}, \quad\left[M_{i}, N_{j}\right]=\imath \epsilon_{i j k} N_{k}, \quad\left[N_{i}, N_{j}\right]=-\imath \epsilon_{i j k} M_{k}
$$

where the three-dimensional rotation generators $M_{i}$ and boosts $N_{i}$ are related with the canonical basis (2.1)-(2.3) as follows

$$
\begin{aligned}
h & =\imath N_{3}, & e_{ \pm} & =\imath\left(N_{1} \pm M_{2}\right), \\
h^{\prime} & =-\imath M_{3}, & e_{ \pm}^{\prime} & =\imath\left( \pm N_{2}-M_{1}\right),
\end{aligned}
$$

and they are Hermitian

$$
M_{i}^{*}=M_{i}, \quad N_{i}^{*}=N_{i} \quad(i=1,2,3) .
$$

It should be stressed that the realization (2.6) of the canonical basis in terms of the physical generators is not unique. Indeed, it is easy to see that the formulas (2.5) are invariant with respect to the cyclic permutation of the indexes $1 \rightarrow 2 \rightarrow 3 \rightarrow 1$ for the generators $M_{i}$ and $N_{i}$. Therefore if we apply such cyclic replacements to the generators of the right side of $((2.6))$ we obtain another physical assignments of the canonical basis. In order to obtain suitable contraction limit (see Sect.4) we perform the cyclic replacements two times and we get other physical assignment of the basis (2.1)-(2.3):

$$
\begin{aligned}
h & =\imath N_{2}, & e_{ \pm} & =\imath\left(N_{3} \pm M_{1}\right), \\
h^{\prime} & =-\imath M_{2}, & e_{ \pm}^{\prime} & =\imath\left( \pm N_{1}-M_{3}\right) .
\end{aligned}
$$

The complete classification of $D=4$ Lorentz quantum algebras in [8] is provided by the following list of the corresponding classical $r$-matrices (see also [12]):

1. Standard $r$-matrices related with realification of Drinfeld-Jimbo deformation of $\mathfrak{s l}(2, \mathbb{C})$ $[13,14,15]$

$$
\begin{aligned}
r_{1}(\alpha, \beta, \gamma) & =\alpha\left(e_{+}^{\prime} \wedge e_{-}+e_{+} \wedge e_{-}^{\prime}\right)+\beta\left(e_{+} \wedge e_{-}-e_{+}^{\prime} \wedge e_{-}^{\prime}\right)+\gamma h \wedge h^{\prime} \\
r_{2}(\alpha) & =\alpha\left(e_{+}^{\prime} \wedge e_{-}+e_{+} \wedge e_{-}^{\prime}+\frac{1}{2} h \wedge h^{\prime}\right) \pm e_{+} \wedge e_{+}^{\prime} .
\end{aligned}
$$

2. Nonstandard $r$-matrices, related with realification of Jordanian deformation of $\mathfrak{s l}(2, \mathbb{C})$ $[16,17,18]$

$$
\begin{aligned}
r_{3}(\alpha, \beta) & =\alpha\left(h \wedge e_{+}-h^{\prime} \wedge e_{+}^{\prime}\right)+\beta e_{+} \wedge e_{+}^{\prime} \\
r_{4}(\alpha) & =\alpha h \wedge e_{+}
\end{aligned}
$$

Further we shall assume that the $r$-matrices are anti-Hermitian, i.e. $r_{i}^{*}=-r_{i}$, therefore all the parameters in (2.9)-(2.12) are purely imaginary.

The standard quantum deformations (2.9) and (2.10) do satisfy modified YB equation and can not be extended to Poincaré algebra [19, 20]. The nonstandard quantum deformations (2.11) and (2.12) can be extended to the whole Poincaré algebra and provide new

\footnotetext{
${ }^{2}$ In what follows the symbol " $"$ means the imaginary unit, $\imath^{2}=-1$.
} 
deformation of relativistic symmetries. Because the quantization of (2.12) by means of the Ogievetsky twist (see [17]) is straightforward and describes the well-known Jordanian deformation, we shall consider here more in detail only the two-parameter deformation generated by the $r$-matrix (2.11).

Further we shall employ as well the complex basis of Lorentz algebra $(\mathfrak{o}(3,1) \simeq$ $\mathfrak{o}(3 ; \mathbb{C}) \oplus \overline{\mathfrak{o}}(3, \mathbb{C}))$ described by two commuting sets of complex generators:

$$
\begin{aligned}
H_{1} & =\frac{1}{2}\left(h+\imath h^{\prime}\right), & E_{1 \pm} & =\frac{1}{2}\left(e_{ \pm}+\imath e_{ \pm}^{\prime}\right), \\
H_{2} & =\frac{1}{2}\left(h-\imath h^{\prime}\right), & E_{2 \pm} & =\frac{1}{2}\left(e_{ \pm}-\imath e_{ \pm}^{\prime}\right),
\end{aligned}
$$

which satisfy the relations (compare with (2.1))

$$
\left[H_{i}, E_{i \pm}\right]= \pm E_{i \pm}, \quad\left[E_{i+}, E_{i-}\right]=2 H_{i} \quad(i=1,2)
$$

where the sets $\left(H_{1}, E_{1 \pm}\right)$ and $\left(H_{2}, E_{2 \pm}\right)$ do commute mutually. The $*$-operation describing the real structure acts on the generators $H_{i}$, and $E_{i \pm}(i=1,2)$ as follows

$$
H_{1}^{*}=-H_{2}, \quad E_{1 \pm}^{*}=-E_{2 \pm}, \quad H_{2}^{*}=-H_{1}, \quad E_{2 \pm}^{*}=-E_{1 \pm} .
$$

The classical $r$-matrix $r_{3}(\alpha, \beta)$ (see (2.11)) in complex basis $(2.13),(2.14)$ takes the form

$$
\begin{gathered}
r_{3}(\alpha, \beta)=r_{3}^{\prime}(\alpha)+r_{3}^{\prime \prime}(\beta) \\
r_{3}^{\prime}(\alpha):=2 \alpha\left(H_{1} \wedge E_{1+}+H_{2} \wedge E_{2+}\right), \quad r_{3}^{\prime \prime}(\beta):=2 i \beta E_{1+} \wedge E_{2+} .
\end{gathered}
$$

\section{Two-parameter nonstandard deformation of $\mathfrak{o}(3,1)$}

The first classical $r$-matrix $r_{3}^{\prime}(\alpha)$ in (2.17) is a sum of two Jordanian classical $r$-matrices $\alpha H_{1} \wedge E_{1}$ and $\alpha H_{2} \wedge E_{2}$ which mutually commute. From this property follows that the first twisting two-tensor $F^{\prime}$ corresponding to the Jordanian type $r$-matrix $r_{3}^{\prime}(\alpha)$ is a product of two Jordanian twists with the same deformation parameter $\alpha$ :

$$
F^{\prime}:=F_{1 J} F_{2 J}=F_{2 J} F_{1 J}=\exp \left(H_{1} \otimes \sigma_{1}+H_{2} \otimes \sigma_{2}\right)
$$

where

$$
\sigma_{i}=\ln \left(1+2 \alpha E_{i+}\right), \quad i=1,2 .
$$

It should be noted that the Jordanian two-tensor (3.1) is $*$-unitary, i.e.

$$
F^{*}=F^{\prime-1}
$$

Since after twisting by the two-tensor (3.1) the generators (3.2) have the primitive coproducts

$$
\Delta^{\left(F^{\prime}\right)}\left(\sigma_{i}\right)=\sigma_{i} \otimes 1+1 \otimes \sigma_{i}, \quad i=1,2
$$


therefore the two-tensor ${ }^{3,4}$

$$
F^{\prime \prime}=\exp \left(\imath \frac{\beta}{2 \alpha^{2}} \sigma_{1} \wedge \sigma_{2}\right)
$$

satisfies the cocycle condition (see [21])

$$
F^{\prime \prime 12}\left(\Delta^{\left(F^{\prime}\right)} \otimes \mathrm{id}\right)\left(F^{\prime \prime}\right)=F^{\prime \prime 23}\left(\mathrm{id} \otimes \Delta^{\left(F^{\prime}\right)}\right)\left(F^{\prime \prime}\right),
$$

and the "unital" normalization condition

$$
(\epsilon \otimes \mathrm{id})\left(F^{\prime \prime}\right)=(\mathrm{id} \otimes \epsilon)\left(F^{\prime \prime}\right)=1 .
$$

Thus the complete twisting two-tensor $F(\alpha, \beta)$ corresponding to the Jordanian type $r$ matrix (2.17) is given as follows

$$
F:=F(\alpha, \beta)=F^{\prime \prime} F^{\prime}=\exp \left(\imath \frac{\beta}{2 \alpha^{2}} \sigma_{1} \wedge \sigma_{2}\right) \exp \left(H_{1} \otimes \sigma_{1}+H_{2} \otimes \sigma_{2}\right) .
$$

Let us express this function in terms of the generators $\left(h, e_{ \pm}, h^{\prime}, e_{ \pm}^{\prime}\right)$. Firstly we notice that the generators $\sigma_{i}$ given by (3.2) do not change after the second twist $F^{\prime \prime}$, i.e. they have the primitive coproducts

$$
\Delta^{(F)}\left(\sigma_{i}\right)=F^{\prime \prime} \Delta^{\left(F^{\prime}\right)}\left(\sigma_{i}\right) F^{\prime \prime-1}=\sigma_{i} \otimes 1+1 \otimes \sigma_{i}, \quad i=1,2 .
$$

Therefore if instead of $\sigma_{1}$ and $\sigma_{2}$ we introduce the new generators $\sigma$ and $\varphi$ by the formulas

$$
\sigma_{1}=\sigma+\imath \varphi, \quad \sigma_{2}=\sigma-\imath \varphi
$$

where

$$
\sigma=\frac{1}{2} \ln \left[\left(1+\alpha e_{+}\right)^{2}+\left(\alpha e_{+}^{\prime}\right)^{2}\right], \quad \varphi=\arctan \frac{\alpha e_{+}^{\prime}}{1+\alpha e_{+}}
$$

then the new generators also have the primitive coproducts

$$
\Delta^{(F)}(\sigma)=\sigma \otimes 1+1 \otimes \sigma, \quad \Delta^{(F)}(\varphi)=\varphi \otimes 1+1 \otimes \varphi,
$$

and moreover they are $*$-Hermitian

$$
\sigma^{*}=\sigma, \quad \varphi^{*}=\varphi .
$$

The formulas inverse to (3.11) have the form

$$
\alpha e_{+}=e^{\sigma} \cos \varphi-1, \quad \alpha e_{+}^{\prime}=e^{\sigma} \sin \varphi .
$$

Substituting (3.10) and (2.13), (2.14) in (3.8) we obtain the following formula for the twist two-tensor in terms of the canonical $\mathfrak{o}(3,1)$-basis $(2.1)-(2.3)$ :

$$
F(\alpha, \beta)=\exp \left(\frac{\beta}{\alpha^{2}} \sigma \wedge \varphi\right) \exp \left(h \otimes \sigma-h^{\prime} \otimes \varphi\right) .
$$

\footnotetext{
${ }^{3}$ In the limit $\alpha \rightarrow 0$ the two-tensor $F^{\prime \prime}$ goes to $\exp \left(2 i \beta E_{1+} \wedge E_{2+}\right)$ which is the twist in the direction of $r_{3}^{\prime \prime}(\alpha=0, \beta)($ see $(2.11))$.

${ }^{4}$ It should be mentioned that modulo the explicit parameters dependence the algebraic form of the twist (3.5) was early pointed by Kulish and Mudrov (see [22]), item 3 on the list on p.6)
} 
This two-tensor is $*$-unitary, i.e.

$$
F^{*}(\alpha, \beta)=F^{-1}(\alpha, \beta)
$$

Deformed coproducts for the canonical $\mathfrak{o}(3,1)$-basis can be obtain in two ways. First way is to apply the twisting two-tensor in the form (3.8) to the trivial coproducts of the complex generators $H_{i}, E_{i}, F_{i}(i=1,2)$ and then by using the formulas (2.13) and (2.14) to derive the coproducts and antipodes for the canonical basis. Other way is to apply the twisting two-tensor in the form (3.15) directly to the trivial coproducts of the canonical generators $(2.1)-(2.2),\left(\Delta_{\alpha, \beta}(\cdot):=F \Delta(\cdot) F^{-1}\right)$. For convenience we shall use the notations: $\mathrm{c}_{\varphi}:=\cos \varphi$ and $\mathrm{s}_{\varphi}:=\sin \varphi$. We obtain the following formulae:

$$
\begin{aligned}
\Delta_{\alpha, \beta}(h)= & h \otimes e^{-\sigma} \mathrm{c}_{\varphi}+1 \otimes h+h^{\prime} \otimes e^{-\sigma} \mathrm{S}_{\varphi}+\frac{\beta}{\alpha^{2}}\left(e^{-\sigma} \mathrm{S}_{\varphi} \otimes e^{-\sigma}\left(\sigma \mathrm{c}_{\varphi}-\varphi \mathrm{s}_{\varphi}\right)\right. \\
& \left.+\left(e^{-\sigma} \mathrm{c}_{\varphi}-1\right) \otimes e^{-\sigma}\left(\varphi \mathrm{c}_{\varphi}+\sigma \mathrm{s}_{\varphi}\right)-\sigma \otimes e^{-\sigma} \mathrm{S}_{\varphi}-\varphi \otimes\left(e^{-\sigma} \mathrm{c}_{\varphi}-1\right)\right), \\
\Delta_{\alpha, \beta}\left(h^{\prime}\right)= & h^{\prime} \otimes e^{-\sigma} \mathrm{c}_{\varphi}+1 \otimes h^{\prime}-h \otimes e^{-\sigma} \mathrm{s}_{\varphi}+\frac{\beta}{\alpha^{2}}\left(-e^{-\sigma} \mathrm{S}_{\varphi} \otimes e^{-\sigma}\left(\sigma \mathrm{S}_{\varphi}+\varphi \mathrm{c}_{\varphi}\right)\right. \\
& \left.+\left(e^{-\sigma} \mathrm{c}_{\varphi}-1\right) \otimes e^{-\sigma}\left(\sigma \mathrm{c}_{\varphi}-\varphi \mathrm{s}_{\varphi}\right)+\varphi \otimes e^{-\sigma} \mathrm{S}_{\varphi}-\sigma \otimes\left(e^{-\sigma} \mathrm{c}_{\varphi}-1\right)\right),
\end{aligned}
$$

The remaining coproducts $\Delta_{\alpha, \beta}\left(e_{-}\right)$and $\Delta_{\alpha, \beta}\left(e_{-}^{\prime}\right)$ are given by lengthy formulae, and therefore we present the $\beta$-dependent terms using the complex basis

$$
\begin{aligned}
& \Delta_{\alpha, \beta}\left(e_{-}\right)=e_{-} \otimes e^{-\sigma} \mathrm{c}_{\varphi}+1 \otimes e_{-}+e_{-}^{\prime} \otimes e^{-\sigma} \mathrm{s}_{\varphi} \\
& +\alpha\left(h \otimes\left(\left\{h^{\prime}, e^{-\sigma} \mathrm{c}_{\varphi}\right\}+\left\{h^{\prime}, e^{-\sigma} \mathrm{s}_{\varphi}\right\}\right)-h^{\prime} \otimes\left(\left\{h^{\prime}, e^{-\sigma} \mathrm{c}_{\varphi}\right\}-\left\{h, e^{-\sigma} \mathrm{s}_{\varphi}\right\}\right)\right. \\
& \left.+\left(h^{2}-h^{\prime 2}\right) \otimes\left(e^{-2 \sigma} \mathrm{c}_{2 \varphi}-e^{-\sigma} \mathrm{c}_{\varphi}\right)+2 h h^{\prime} \otimes\left(e^{-2 \sigma} \mathrm{s}_{2 \varphi}-e^{-\sigma} \mathrm{s}_{\varphi}\right)\right) \\
& +\frac{\imath \beta}{4 \alpha}\left(\mathcal{E}_{1}+\mathcal{E}_{1}^{*}\right)-\frac{\beta^{2}}{4 \alpha^{3}}\left(\mathcal{E}_{2}+\mathcal{E}_{2}^{*}\right), \\
& \Delta_{\alpha, \beta}\left(e_{-}^{\prime}\right)=e_{-}^{\prime} \otimes e^{-\sigma} \mathrm{c}_{\varphi}+1 \otimes e_{-}^{\prime}+e_{-} \otimes e^{-\sigma} \mathrm{s}_{\varphi} \\
& +\alpha\left(h^{\prime} \otimes\left(\left\{h, e^{-\sigma} \mathrm{c}_{\varphi}\right\}+\left\{h^{\prime}, e^{-\sigma} \mathrm{s}_{\varphi}\right\}\right)+h \otimes\left(\left\{h^{\prime}, e^{-\sigma} \mathrm{c}_{\varphi}\right\}-\left\{h, e^{-\sigma} \mathrm{s}_{\varphi}\right\}\right)\right. \\
& \left.-\left(h^{2}-h^{\prime 2}\right) \otimes\left(e^{-2 \sigma} \mathrm{s}_{2 \varphi}-e^{-\sigma} \mathrm{s}_{\varphi}\right)+2 h h^{\prime} \otimes\left(e^{-2 \sigma} \mathrm{c}_{2 \varphi}-e^{-\sigma} \mathrm{c}_{\varphi}\right)\right) \\
& +\frac{\beta}{4 \alpha}\left(\mathcal{E}_{1}-\mathcal{E}_{1}^{*}\right)+\frac{\imath \beta^{2}}{4 \alpha^{3}}\left(\mathcal{E}_{2}-\mathcal{E}_{2}^{*}\right),
\end{aligned}
$$

where

$$
\begin{gathered}
\mathcal{E}_{1}=\left\{\tilde{H}_{1}, e^{-\sigma_{1}}\right\} \otimes \sigma_{2} e^{-\sigma_{1}}-\sigma_{2} \otimes\left\{\tilde{H}_{1}, e^{-\sigma_{1}}\right\}+\Lambda_{1} \otimes\left\{\tilde{H}_{1}, e^{-\sigma_{1}}\right\} \sigma_{2} \\
-\left\{\tilde{H}_{1}, \Lambda_{1}\right\} \otimes \sigma_{2} \Lambda_{1} e^{-\sigma_{1}}-\left\{\tilde{H}_{1}, \sigma_{2}\right\} \otimes \Lambda_{1} e^{-\sigma_{1}}, \\
\mathcal{E}_{2}=\sigma_{2}^{2} \otimes \Lambda_{1} e^{-\sigma_{1}}+\Lambda_{1} e^{-\sigma_{1}} \otimes \sigma_{2}^{2} e^{-\sigma_{1}}+\Lambda_{1}^{2} \otimes \sigma_{2}^{2} \Lambda_{1} e^{-\sigma_{1}}-2 \Lambda_{1} \sigma_{2} \otimes \sigma_{2} \Lambda_{1} e^{-\sigma_{1}},
\end{gathered}
$$


and

$$
\tilde{H}_{1}=h+\imath h^{\prime}, \quad \Lambda_{1}=e^{-\sigma_{1}}-1, \quad \sigma_{1}=\sigma+\imath \varphi, \quad \sigma_{2}=\sigma-\imath \varphi .
$$

Here the brackets $\{\cdot, \cdot\}$ mean the anti-commutator $\{a, b\}=a b+b a$.

Explicit formulae for the antipodes are given by

$$
\begin{gathered}
S_{\alpha, \beta}(\sigma)=-\sigma, \quad S_{\alpha, \beta}(\varphi)=-\varphi \\
S_{\alpha, \beta}(h)=-h e^{\sigma} \mathrm{c}_{\varphi}+h^{\prime} e^{\sigma} \mathrm{s}_{\varphi}, \quad S_{\alpha, \beta}\left(h^{\prime}\right)=-h^{\prime} e^{\sigma} \mathrm{c}_{\varphi}-h e^{\sigma} \mathrm{s}_{\varphi}, \\
S_{\alpha, \beta}\left(e_{-}\right)=-e_{-} e^{\sigma} \mathrm{c}_{\varphi}+e_{-}^{\prime} e^{\sigma} \mathrm{s}_{\varphi}+\alpha\left(h^{2}-h^{\prime 2}\right)\left(e^{2 \sigma} \mathrm{c}_{2 \varphi}+e^{\sigma} \mathrm{c}_{\varphi}\right) \\
-2 \alpha h h^{\prime}\left(e^{2 \sigma} \mathrm{s}_{2 \varphi}+e^{\sigma} \mathrm{s}_{\varphi}\right) \\
S_{\alpha, \beta}\left(e_{-}^{\prime}\right)=-e_{-}^{\prime} e^{\sigma} \mathrm{c}_{\varphi}-e_{-} e^{\sigma} \mathrm{s}_{\varphi}+\alpha\left(h^{2}-h^{\prime 2}\right)\left(e^{2 \sigma} \mathrm{s}_{2 \varphi}+e^{\sigma} \mathrm{s}_{\varphi}\right) \\
+2 \alpha h h^{\prime}\left(e^{2 \sigma} \mathrm{c}_{2 \varphi}+e^{\sigma} \mathrm{c}_{\varphi}\right) .
\end{gathered}
$$

Using the relations (2.6) the formulae (3.17)-(3.27) can be rewritten in terms of physical generators $M_{j}$ and $N_{j}(j=1,2,3)$.

\section{Extension of the deformation to Poincaré algebra}

The $D=4$ Lorentz algebra (2.6) can be extended to $D=4$ Poincaré algebra by adding the mutually commuting four-momentum operators $\left(P_{0}, P_{1}, P_{2}, P_{3}\right)$ satisfying the relations $(j, k, l=1,2,3)$

$$
\begin{array}{ll}
{\left[M_{i}, P_{j}\right]=\imath \epsilon_{i j k} P_{k},} & {\left[M_{i}, P_{0}\right]=0,} \\
{\left[N_{i}, P_{j}\right]=-\imath \delta_{i j} P_{0},} & {\left[N_{i}, P_{0}\right]=-\imath P_{i} .}
\end{array}
$$

The formulas (4.1) say that a linear span of the four-momentum generators $P_{\mu}, \quad(\mu=$ $0,1,2,3)$ is a four-dimensional $\mathfrak{o}(3,1)$-module with respect to the adjoint action of Lorentz algebra on the four-momentum space. In order to describe in this space the actions of the canonical basis $\left(h, e_{ \pm}, h^{\prime}, e_{ \pm}^{\prime}\right)$ it is useful to use as a technical tool (see also [9]) the matrix realization of the four-momenta. The matrix realization is constructed as follows.

First we introduce the following basis in the four-momentum space

$$
\begin{aligned}
P_{11} & =\frac{1}{2}\left(P_{0}+P_{3}\right), & P_{12} & =\frac{1}{2}\left(P_{1}-\imath P_{2}\right), \\
P_{21} & =\frac{1}{2}\left(P_{1}+\imath P_{2}\right), & P_{22} & =\frac{1}{2}\left(P_{0}-P_{3}\right) .
\end{aligned}
$$

Let $\hat{P}_{i j},(i, j=1,2)$ be the $2 \times 2$-matrices $\left(\hat{P}_{i j}\right)_{k l}=\delta_{i k} \delta_{j l}$. The matrix realization of the four-momenta is given by $P_{i j} \rightarrow \hat{P}_{i j}$. The arbitrary four-vector $\mathbf{P}:=\sum_{i, j=1,2} \xi_{i j} P_{i j}$, $\left(\xi_{i j} \in \mathbb{R}\right)$, is represented by the general $2 \times 2$-matrix

$$
\mathbf{P} \rightarrow \hat{\mathbf{P}}:=\sum_{i, j=1,2} \xi_{i j} \hat{P}_{i j}=\left(\begin{array}{ll}
\xi_{11} & \xi_{12} \\
\xi_{21} & \xi_{22}
\end{array}\right),
$$


which will be used below to describe the transformation properties of the generators (4.2) in compact way. The corresponding $2 \times 2$-matrix realization of the Lorentz algebra (2.1)-(2.3) will be used

$$
\begin{aligned}
h \rightarrow H & =\frac{1}{2}\left(\begin{array}{rr}
1 & 0 \\
0 & -1
\end{array}\right), \quad e_{+} \rightarrow E_{+}=\left(\begin{array}{ll}
0 & 1 \\
0 & 0
\end{array}\right), \quad e_{-} \rightarrow E_{-}=\left(\begin{array}{ll}
0 & 0 \\
1 & 0
\end{array}\right), \\
h^{\prime} & \rightarrow H^{\prime}=\imath H, \quad e_{+}^{\prime} \rightarrow E_{+}^{\prime}=\imath E_{+}, \quad e_{-}^{\prime} \rightarrow E_{-}^{\prime}=\imath E_{-} .
\end{aligned}
$$

The action of any element $x \in\left(h, e_{ \pm}, h^{\prime}, e_{ \pm}^{\prime}\right)$ on the matrix-vector $\hat{\mathbf{P}}$ is given by (see [9]):

$$
[x, \mathbf{P}] \rightarrow x \triangleright \hat{\mathbf{P}}:=X \hat{\mathbf{P}}+\hat{\mathbf{P}} X^{+} .
$$

where $X^{+}$is the hermitian conjugate of $X$. Using the formula (4.6) we find, for example,

$$
\begin{array}{cc}
h \triangleright \hat{\mathbf{P}}=\left(\begin{array}{cc}
\xi_{11} & 0 \\
0 & -\xi_{22}
\end{array}\right), & h^{\prime} \triangleright \hat{\mathbf{P}}=\imath\left(\begin{array}{cc}
0 & \xi_{21} \\
-\xi_{12} & 0
\end{array}\right), \\
e_{+} \triangleright \hat{\mathbf{P}}=\left(\begin{array}{cc}
\xi_{21}+\xi_{12} & \xi_{22} \\
\xi_{22} & 0
\end{array}\right), & e_{+}^{\prime} \triangleright \hat{\mathbf{P}}=\imath\left(\begin{array}{cc}
\xi_{21}-\xi_{12} & \xi_{22} \\
-\xi_{22} & 0
\end{array}\right) .
\end{array}
$$

The classical $r$-matrix (2.10) for $D=4$ Lorentz algebra satisfies classical YB equation and provides also the deformation of $D=4$ Poincaré algebra. The modification of classical Hopf algebra structure is described by the twist function (3.15). Let us calculate the twisted coproducts of the four-momenta in our $2 \times 2$-matrix realization (4.3) using the restricted twist two-tensor $F^{\prime}(\alpha):=F(\alpha, \beta=0)$. We have

$$
\Delta_{\alpha}(\hat{\mathbf{P}})=F^{\prime}(\alpha) \hat{\mathbf{P}} \otimes 1 F^{\prime-1}(\alpha)+F^{\prime}(\alpha) 1 \otimes \hat{\mathbf{P}} F^{\prime-1}(\alpha) .
$$

By employing the formulas (4.6)-(4.8) we find

$$
\begin{aligned}
\Delta_{\alpha}(\hat{\mathbf{P}})= & \left(\begin{array}{cc}
\xi_{11} \otimes e^{\sigma} & \xi_{12} \otimes e^{\imath \varphi} \\
\xi_{21} \otimes e^{-\imath \varphi} & \xi_{22} \otimes e^{-\sigma}
\end{array}\right)+1 \otimes\left(\begin{array}{ll}
\xi_{11} & \xi_{12} \\
\xi_{21} & \xi_{22}
\end{array}\right) \\
& +\alpha h \otimes\left\{e^{-\sigma+\imath \varphi}\left(\begin{array}{cc}
\xi_{21} & \xi_{22} \\
0 & 0
\end{array}\right)+e^{-\sigma-\imath \varphi}\left(\begin{array}{ll}
\xi_{12} & 0 \\
\xi_{22} & 0
\end{array}\right)\right\} \\
& -\imath \alpha h^{\prime} \otimes\left\{e^{-\sigma+\imath \varphi}\left(\begin{array}{cc}
\xi_{21} & \xi_{22} \\
0 & 0
\end{array}\right)-e^{-\sigma-\imath \varphi}\left(\begin{array}{ll}
\xi_{12} & 0 \\
\xi_{22} & 0
\end{array}\right)\right\} \\
& +\alpha^{2}\left(h^{2}+h^{\prime 2}\right) \otimes e^{-2 \sigma}\left(\begin{array}{cc}
\xi_{22} & 0 \\
0 & 0
\end{array}\right) .
\end{aligned}
$$

Because $\partial \hat{\mathbf{P}} / \partial \xi_{i j}=\hat{P}_{i j}$, by differentiating (4.10) we obtain the formulas for the twisted coproducts of the four-momentum components $\hat{P}_{i j}$. These formulas after replacing the 
realizations $\hat{P}_{i j}$ by the initial generators $P_{i j}$ take the following compact form:

$$
\begin{aligned}
\left(\begin{array}{cc}
\Delta_{\alpha}\left(P_{11}\right) & \Delta_{\alpha}\left(P_{12}\right) \\
\Delta_{\alpha}\left(P_{21}\right) & \Delta_{\alpha}\left(P_{22}\right)
\end{array}\right)= & \left(\begin{array}{cc}
P_{11} \otimes e^{\sigma} & P_{12} \otimes e^{-\imath \varphi} \\
P_{21} \otimes e^{\imath \varphi} & P_{22} \otimes e^{-\sigma}
\end{array}\right)+1 \otimes\left(\begin{array}{cc}
P_{11} & P_{12} \\
P_{21} & P_{22}
\end{array}\right) \\
& +\alpha h \otimes\left(\begin{array}{cc}
0 & e^{-\sigma-\imath \varphi} P_{11} \\
e^{-\sigma+\imath \varphi} P_{11} & e^{-\sigma+\imath \varphi} P_{12}+e^{-\sigma-\imath \varphi} P_{21}
\end{array}\right) \\
& -\imath \alpha h^{\prime} \otimes\left(\begin{array}{cc}
0 & -e^{-\sigma-\imath \varphi} P_{11} \\
e^{-\sigma+\imath \varphi} P_{11} & e^{-\sigma+\imath \varphi} P_{12}-e^{-\sigma-\imath \varphi} P_{21}
\end{array}\right) \\
& +\alpha^{2}\left(h^{2}+h^{\prime 2}\right) \otimes\left(\begin{array}{cc}
0 & 0 \\
0 & e^{-2 \sigma} P_{11}
\end{array}\right) .
\end{aligned}
$$

In analogous way we can calculate the complete twisted coproducts of the four-momenta using the general twist two-tensor $F(\alpha, \beta)$, but these formulae are lengthly.

\section{Two-parameter light-cone deformation of the $D=3$ Poincaré algebra}

The $D=4$ Lorentz algebra $\mathfrak{o}(3,1)$ (see $(2.5)$ ) can be reinterpreted as describing the $D=3$ de-Sitter algebra where the generators $N_{1}, N_{2}$ describe two $D=3$ boosts, $M_{3}$ generates $\mathfrak{o}(2)$ rotations and three curved translations are generated by $M_{1}, M_{2}$ and $N_{3}$. Therefore the formulae (3.17)-(3.25) can be used for the description of the quantum Hopf-algebraic deformations of $D=3$ de-Sitter algebra. Below we shall use the formulae (2.8) relating the physical Lorentz generators with the mathematical basis (2.1)-(2.3). In this realization the classical $r$-matrix (2.11) has the form

$$
r_{3}(\alpha, \beta)=-\alpha\left(N_{2} \wedge\left(N_{3}+M_{1}\right)+M_{2} \wedge\left(N_{1}-M_{3}\right)\right)-\beta\left(N_{3}+M_{1}\right) \wedge\left(N_{1}-M_{3}\right) .
$$

Introducing the standard dS-rescaling $M_{1}=R \mathcal{P}_{2}, M_{2}=-R \mathcal{P}_{1}, N_{3}=R \mathcal{P}_{0}$ we obtain for the $r$-matrix (5.1) the following form

$$
r_{3}(\alpha, \beta)=-\alpha R\left(N_{2} \wedge\left(\mathcal{P}_{0}+\mathcal{P}_{2}\right)+\mathcal{P}_{1} \wedge\left(M_{3}-N_{1}\right)\right)-\beta R\left(\mathcal{P}_{0}+\mathcal{P}_{2}\right) \wedge\left(M_{3}-N_{1}\right) .
$$

Now we put $\alpha=-\imath / \kappa R, \beta=-\imath / \kappa^{\prime} R$ (where $\kappa$ and $\kappa^{\prime}$ are real masslike parameters) and perform the limit $R \rightarrow \infty$. In such a way we obtain the classical $r$-matrix for the $D=3$ Poincaré algebra $\left(\lim _{R \rightarrow \infty} \mathcal{P}_{\mu}=P_{\mu}, \mu=0,1,2\right)$ :

$$
\begin{aligned}
r_{\kappa, \kappa^{\prime}} & :=\lim _{R \rightarrow \infty} r_{3}\left(\frac{-\imath}{\kappa R}, \frac{-\imath}{\kappa^{\prime} R}\right) \\
& =\frac{\imath}{\kappa}\left(N_{2} \wedge\left(P_{0}+P_{2}\right)+P_{1} \wedge\left(M_{3}-N_{1}\right)\right)+\frac{\imath}{\kappa^{\prime}}\left(P_{0}+P_{2}\right) \wedge\left(M_{3}-N_{1}\right),
\end{aligned}
$$

where the parameters $\kappa$ and $\kappa^{\prime}$ are new dimensionfull deformation parameters. The $D=3$

Poincaré algebra is described by the generators $M_{3}, N_{1}, N_{2}$ of the $D=3$ Lorentz algebra $\mathfrak{o}(2,1)$, and the generators $P_{0}, P_{1}, P_{2}$ span the threemomentum sector. 
The classical $r$-matrix (5.3) describes two-parameter light cone $\kappa$-deformation of the $D=3$ Poincaré algebra ${ }^{5}$. Using the commutation relations for the generators $M_{3}, N_{1}, N_{2}$, $P_{ \pm}:=P_{0} \pm P_{2}$ it easy to check that the $r$-matrix $r_{\kappa, \kappa^{\prime}}$, when $\kappa^{\prime}$ goes to $\infty$, is of Jordanian type ${ }^{6}$. Therefore using known general formulae (see [24, 25]) we can immediately write down the twisting two-tensor corresponding to the $r$-matrix $r_{\kappa, \kappa^{\prime}}$. However we can obtain also a twisting two-tensor corresponding to the classical $r$-matrix (5.3) by applying the $\mathrm{dS}$ contraction limit to the full twisting two-tensor (3.15). First of all it is easy to obtain in the limit $R \rightarrow \infty$ the following formulas:

$$
\begin{gathered}
\sigma_{+}:=\lim _{R \rightarrow \infty} \sigma=\lim _{R \rightarrow \infty} \frac{1}{2} \ln \left[\left(1+\frac{1}{\kappa} \mathcal{P}_{+}\right)^{2}+\frac{1}{\kappa^{2} R^{2}}\left(N_{1}+M_{3}\right)^{2}\right]=\ln \left(1+\frac{1}{\kappa} P_{+}\right), \\
\lim _{R \rightarrow \infty}(R \varphi)=\lim _{R \rightarrow \infty} R \cdot \arctan \frac{\left(N_{1}-M_{3}\right) / \kappa R}{\left(1+\mathcal{P}_{+} / \kappa\right)}=\frac{1}{\kappa}\left(N_{1}-M_{3}\right) e^{-\sigma_{+} .}
\end{gathered}
$$

Using the formulas (5.4) and (5.5) we get the twist two-tensor $F_{\kappa, \kappa^{\prime}}$ corresponding the classical $r$-matrix (5.3)

$$
\begin{aligned}
F_{\kappa, \kappa^{\prime}}=\lim _{R \rightarrow \infty} F\left(\frac{-\imath}{\kappa R}, \frac{-\imath}{\kappa^{\prime} R}\right): & =\exp \left(\frac{\imath \kappa}{\kappa^{\prime}} \sigma_{+} \otimes\left(N_{1}-M_{3}\right) e^{-\sigma_{+}}\right) \\
& \times \exp \left(\frac{\imath}{\kappa} P_{1} \otimes\left(N_{1}-M_{3}\right) e^{-\sigma_{+}}\right) \exp \left(\imath N_{2} \otimes \sigma_{+}\right) .
\end{aligned}
$$

By applying the rescaling of the formulas (3.17)-(3.22) (introducing dS radius $R$ and performing the limit $R \rightarrow \infty$ ) we obtain the list of deformed co-products for all generators of the $D=3$ Poincaré algebra. For convenience in what follows we set $\Lambda_{+}:=e^{-\sigma_{+}}-1$, $L_{+}:=N_{1}-M_{+}$and $L_{-}:=N_{1}+M_{+}$. The coproducts are given by

$$
\begin{aligned}
\Delta_{\kappa, \kappa^{\prime}}\left(\sigma_{+}\right)= & \sigma_{+} \otimes 1+1 \otimes \sigma_{+} \\
\Delta_{\kappa, \kappa^{\prime}}\left(P_{1}\right)= & P_{1} \otimes e^{-\sigma_{+}}+1 \otimes P_{1}+\frac{\kappa^{2}}{\kappa^{\prime}}\left(\Lambda_{+} \otimes \sigma_{+} e^{-\sigma_{+}}-\sigma_{+} \otimes \Lambda_{+}\right), \\
\Delta_{\kappa, \kappa^{\prime}}\left(P_{-}\right)= & P_{-} \otimes e^{-\sigma_{+}}+1 \otimes P_{-}-\frac{1}{\kappa}\left(2 P_{1} \otimes P_{1} e^{-\sigma_{+}}+P_{1}^{2} \otimes \Lambda_{+} e^{-\sigma_{+}}\right) \\
& -\frac{2 \kappa}{\kappa^{\prime}}\left(P_{1} e^{-\sigma_{+}} \otimes \sigma_{+} e^{-\sigma_{+}}-\sigma_{+} \otimes P_{1} e^{-\sigma_{+}}-P_{1} \Lambda_{+} \otimes \sigma_{+} \Lambda_{+} e^{-\sigma_{+}}\right. \\
& \left.-P_{1} \sigma_{+} \otimes \Lambda_{+} e^{-\sigma_{+}}+\Lambda_{+} \otimes P_{1} e^{-\sigma_{+}}\right)-\frac{\kappa^{3}}{\kappa^{\prime 2}}\left(\Lambda_{+} e^{-\sigma_{+}} \otimes \sigma_{+}^{2} e^{-\sigma_{+}}\right. \\
& \left.+\sigma_{+}^{2} \otimes \Lambda_{+} e^{-\sigma_{+}}+\Lambda_{+}^{2} \otimes \sigma_{+}^{2} \Lambda_{+} e^{-\sigma_{+}}-2 \Lambda_{+} \sigma_{+} \otimes \sigma_{+} \Lambda_{+} e^{-\sigma_{+}}\right), \\
\Delta_{\kappa, \kappa^{\prime}}\left(L_{+}\right)= & L_{+} \otimes e^{\sigma_{+}}+e^{\sigma_{+}} \otimes L_{+}, \\
\Delta_{\kappa, \kappa^{\prime}}\left(N_{2}\right)= & N_{2} \otimes e^{-\sigma_{+}}+1 \otimes N_{2}+\frac{1}{\kappa} P_{1} \otimes L_{+} e^{-2 \sigma_{+}}-\frac{\kappa}{\kappa^{\prime}}\left(L_{+} e^{-\sigma_{+}} \otimes \Lambda_{+}\right. \\
-L_{+} & \left.e^{-2 \sigma_{+}} \otimes \sigma_{+} e^{-\sigma_{+}}-\Lambda_{+} \otimes L_{+}\left(\sigma_{+}+1\right) e^{-2 \sigma_{+}}+\sigma_{+} \otimes L_{+} e^{-2 \sigma_{+}}\right),
\end{aligned}
$$

\footnotetext{
${ }^{5}$ For analogous considerations for the $D=4$ case see [23].

${ }^{6}$ Compare with [24], formulae (2.12), after assignment $h_{\theta}=i N_{2}, e_{\theta}=P_{+}, e_{\gamma_{1}}=P_{1}, e_{\gamma_{-1}}=\left(M_{3}-N_{1}\right)$ (see also [23]).
} 
The last coproduct appears very lengthly and we present it only in the limit $\kappa^{\prime} \rightarrow \infty$ :

$$
\begin{aligned}
\Delta_{\kappa}\left(L_{-}\right)= & L_{-} \otimes e^{-\sigma_{+}}+1 \otimes L_{-}+\frac{1}{\kappa}\left(P_{-} \otimes L_{+} e^{-\sigma_{+}}-\right. \\
& \left.-P_{1} \otimes\left\{N_{2}, e^{-\sigma_{+}}\right\}-2 N_{2} \otimes P_{1} e^{-\sigma_{+}}-2 N_{2} P_{1} \otimes \Lambda_{+} e^{-\sigma_{+}}\right) \\
& -\frac{1}{\kappa^{2}}\left(P_{1} \otimes\left\{P_{1}, L_{+}\right\} e^{-2 \sigma_{+}}+P_{1}^{2} \otimes L_{+}\left(2 \Lambda_{+}+1\right) e^{-2 \sigma_{+}}\right) .
\end{aligned}
$$

Using the formulas (3.23)-(3.27) one can calculate the antipodes

$$
\begin{gathered}
S_{\kappa, \kappa^{\prime}}\left(\sigma_{+}\right)=-\sigma_{+}, \quad S_{\kappa, \kappa^{\prime}}\left(P_{1}\right)=-P_{1} e^{\sigma_{+}}, \\
S_{\kappa, \kappa^{\prime}}\left(P_{-}\right)=-P_{-} e^{\sigma_{+}}-\frac{1}{\kappa} P_{1}^{2}\left(e^{2 \sigma_{+}}+e^{\sigma_{+}}\right), \\
S_{\kappa, \kappa^{\prime}}\left(L_{+}\right)=-L_{+} e^{-2 \sigma_{+}}, \quad S_{\kappa, \kappa^{\prime}}\left(N_{2}\right)=-N_{2} e^{\sigma_{+}}+\frac{1}{\kappa} P_{1} L_{+}, \\
S_{\kappa, \kappa^{\prime}}\left(L_{-}\right)=-L_{-} e^{\sigma_{+}}-\frac{1}{\kappa}\left(P_{-} L_{+}+2 N_{2} P_{1}\left(e^{2 \sigma_{+}}+e^{\sigma_{+}}\right)\right)+\frac{1}{\kappa^{2}} P_{1}^{2} L_{+}\left(2 e^{\sigma_{+}}+1\right) .
\end{gathered}
$$

\section{Outlook}

In this paper we have described firstly in detail the Hopf algebra structure of the twoparameter twisted $D=4$ Lorentz algebras, which induce as well the twist quantization of the Poincaré algebra. It should be stressed that contrary to the cases of twisted Lorentz and Poincaré symmetries with Abelian twists which were considered recently in the literature $[3,4,5,6,7]$ our deformation is generated by the non-Abelian twist. We also performed the dS contraction limit of the twisted $D=4$ Lorentz algebra and obtained the deformation of the $D=3$ Poincaré algebra with the explicit Hopf algebra structure.

A question which should be further addressed is the Hopf structure of dual quantum Poincaré group and the description of corresponding noncommutative Minkowski space. The quantum Poincaré groups generated by the classical Lorentz $r$-matrix (2.11) can be found on the list of deformed Poincaré groups given by Podleś and Woronowicz [20]. Other method providing a quantum algebra of noncommutative Poincaré group parameters is to calculate in adjoint $4 \times 4$ matrix representation the quantum $R$-matrix and apply the RTT method [26]. In our case the universal $R$-matrix takes the form

$$
\begin{aligned}
R=F^{21}(\alpha, \beta) F^{-1}(\alpha, \beta)= & \exp \left(\frac{\beta}{\alpha^{2}} \varphi \wedge \sigma\right) \exp \left(\sigma \otimes h-\varphi \otimes h^{\prime}\right) \\
& \times \exp \left(h^{\prime} \otimes \varphi-h \otimes \sigma\right) \exp \left(\frac{\beta}{\alpha^{2}} \varphi \wedge \sigma\right) .
\end{aligned}
$$

Using the adjoint matrix representation of Lorentz generators $\left(M_{i j}\right)_{\mu \nu}=\delta_{\mu i} \delta_{\nu j}-\delta_{\mu j} \delta_{\nu i}$, $\left(M_{0 j}\right)_{\mu \nu}=\delta_{\mu 0} \delta_{\nu j}-\delta_{\mu j} \delta_{\nu 0}(\mu, \nu=0,1,2,3 ; i, j=1,2,3)$ we can obtain from (5.1) a $16 \times 16$ dimensional quantum adjoint $R$-matrix for the Lorentz group. The $R$-matrix (6.1) can 
be treated also as the universal $R$-matrix for $D=4$ Poincaré algebra, and one can obtain easily the $25 \times 25$-dimensional quantum $R$-matrix for $D=4$ Poincaré algebra ${ }^{7}$.

It is well-known that one can obtain the $D=4 \kappa$-deformed Poincaré algebra in its standard form (with time variable quantized) if we perform the quantum AdS contraction of $q$-deformed AdS algebra $U_{q}(\mathfrak{s o}(3,2))$ (see [11]). In Sect.5 we have shown that if we treat the $D=4$ Lorentz algebra as the de-Sitter algebra one obtains by the suitable quantum dS contraction the generalized light cone $\kappa$-deformation of the $D=3$ Poincaré algebra, with two independent masslike parameters $\kappa$ and $\kappa^{\prime}$. Therefore one can conclude that quantum extension of Inonu-Wigner contraction procedure to the twisted de-Sitter (or anti-de-Sitter) Hopf algebras can be used as the derivation method of twisted Poincaré symmetries.

\section{Acknowledgments}

The paper has been supported by KBN grant 1PO3B01828 (A.B., J.L.) and the grants RFBR-05-01-01086, INTAS-OPEN-03-51-3350 (V.N.T.). The third author would like to thank Institute for Theoretical Physics, University of Wrocław for hospitality.

\section{References}

[1] S. Doplicher, K. Fredenhagen, and J.E. Roberts, Phys. Lett., B331, 39 (1994); Commun. Math. Phys. 172, 187 (1995); hep-th/0303037.

[2] N. Seiberg and E. Witten, JHEP, 9909, 032 (1999); hep-th/9908142.

[3] J. Wess, Proc. of 2003 Workshop in Vrnacha Banya, Serbia, Aug. 2003; publ. Belgrad, 2004, p. 122; hep-th/0408080.

[4] M. Chaichian, P.P. Kulish, K. Nishijima, and A. Tureanu, Phys. Lett., B604, 98 (2004); hep-th/0408069.

[5] P. Aschieri, Ch. Blohmann, M. Dimitrijevic, F. Meyer, P. Schupp, and J. Wess, Class. Quant. Grav., 22, 3511 (2005); hep-th/0504183.

[6] J. Lukierski and M. Woronowicz, Phys. Lett., B633, 116 (2006); hep-th/0508083.

[7] C. Gonera, P. Kosinski, P. Maslanka, and S. Giller, Phys.Lett., B622, 192 (2005); hep-th/0504132.

[8] S. Zakrzewski, Lett. Math. Phys., 32, 11 (1994).

[9] S. Zakrzewski, Commun. Math. Phys., 187, 285 (1997); http://arxiv.org/abs/q-al/9602001.

[10] A. Borowiec, J. Lukierski, and V.N. Tolstoy, Czech. J. Phys., 55, 11 (2005); http://xxx.lanl.gov/abs/hep-th/0301033.

\footnotetext{
${ }^{7}$ For that purpose one should insert in (6.1) the $5 \times 5$-dimensional realization of Poincaré algebra, with the momenta generators $P_{\mu}$ described by the matrices having only the fifth column nonvanishing $\left(P_{\mu}=\delta_{\mu 5}\right)$.
} 
[11] J. Lukierski, A. Nowicki, H. Ruegg, and V.N. Tolstoy, Phys. Lett., B264, 331 (1991).

[12] S.L. Woronowicz and S. Zakrzewski, Comp. Math., 90, 211 (1994).

[13] V.G. Drinfeld, in Proc. of XX-th Int. Math. Congress (1986), Berkeley (USA), p. 1986.

[14] M. Jimbo, Lett. Math. Phys., 10, 63 (1985).

[15] W.B. Schmidke, J. Wess, and B. Zumino, Z. Phys., C52, 471 (1991).

[16] C. Ohn, Lett. Math. Phys., 25, 85 (1992).

[17] O.V. Ogievetsky, Suppl. Rend. Circ. Math. Palermo, Serie II, 37, 185 (1993); preprint MPI-Ph/92-99 (1992).

[18] B. Abdesselam, A. Chakrabarti, R. Chakrabarti, and J. Segar, http://arxiv.org/abs/q-alg/9807100.

[19] S. Majid, J. Math. Phys., 34, 2045 (1993).

[20] P. Podles and S.L. Woronowicz, Proc. of First Carribian Spring School of Mathematics and Theoretical Physics, June 1993, eds: R. Coquereaux, M. Dubois-Violette and P. Flad, Scientific, p. 364 (1995).

[21] V.G. Drinfeld, Leningrad Math. J., 1, 1419 (1990).

[22] P. Kulish and A. Mudrov, Proc. Steklov Inst. Math., 226, 97 (1999); http: //xxx.lanl.gov/abs/math.QA/9901019.

[23] A. Borowiec, J. Lukierski, and V.N. Tolstoy, Eur. Phys. J., C44, 139 (2005); arXiv:hep-th/0412131.

[24] V.N. Tolstoy, in: Proc. of International Workshop "Supersymmetries and Quantum Symmetries (SQS'03)", Russia, Dubna, July, 2003, eds: E. Ivanov and A. Pashnev, publ. JINR, Dubna, p. 242 (2004); http://xxx.lanl.gov/abs/math.QA/0402433.

[25] P.P. Kulish, V.D. Lyakhovsky and A. Mudrov, J. Math. Phys., 24, 4569 (1999).

[26] L.D. Faddeev, N.Yu. Reshetikhin, and L.A. Takhtadjan, Algebra i Analiz, 1, 178 (1989). 